\title{
Current Transport at the Atomic Scale**
}

\author{
By Ursula Schröter, ${ }^{\star}$ Elke Scheer, Rainer Arnold, Cecile Bacca, Tobias Böhler, \\ Jochen Grebing, Patrick Konrad, Vojko Kunej, Ning Kang, Hans-Fridtjof Pernau, \\ and Christian Schirm
}

Electrical contacts of the width of only one atom can be realized by the break-junction technique. The conductance decreases stepwise due to structural reconfigurations when tearing a nano-bridge in the fewatom range. Transport is described by an ensemble of channels with possibly quite high transmission probabilities. For a single break-junction the last one-atom contact consists of a material-specific channel ensemble, determined by the chemical valance as verified for quite a number of metals. d-electrons in halfmetals and spin-effects in magnetic materials will complicate this simple model. Break-junctions also provide ideal contacts to investigate transport through freely suspended clusters or molecules like DNA.

\section{Introduction}

The in principle thinnest diameter of a conductor resp. the smallest possible contact between two conductors consists of one atom. It is indeed possible to realize such contacts, and current transport through them differs fundamentally from that in macroscopic conductors and also from the behaviour of electron gases in restricted geometries. The atomic properties of the central particle as well as the embedding in its surroundings play a role.

\section{Realization of One-Atom-Contacts and Measurement Setup}

One-atom-contacts are feasible by pulling free-standing metallic nano-bridges. For sample preparation a polished bronze wafer is covered by an isolation and sacrificial layer of polyimid of a few $\mu \mathrm{m}$. Bronze is used, because it stays flexible even at low temperatures. On top of the polyimid layer, a double resist layer of MMA and PMMA is spincoated, into which the structure of a stripe with contact pads and an approximately $100 \mathrm{~nm}$ wide constriction is written by e-beam lithography. Development in MIBK removes the exposed parts of the resist. The double resist layer with the easier solvable MMA underneath is used in order to produce sufficient undercut for the following lift-off process. With a thermal or

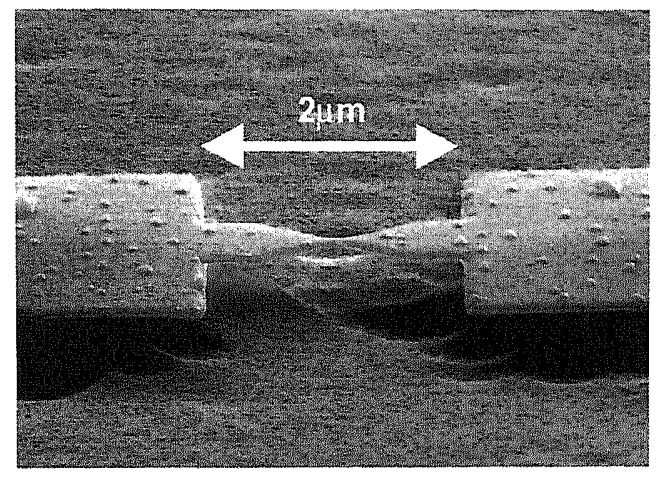

Fig. 1. Electron-microscope image of aluminium break junction. (Courtesy of E. Scheer)

e-beam evaporator an around $100 \mathrm{~nm}$ thick layer of the metal to be investigated is deposited. Because of the shadowing effect of the resist edges, the metal thickness in the constriction becomes somewhat less, though. The unexposed parts of the resist are then removed in aceton (lift-off), such that only the metal

[*] Dr. U. Schröter, Dr. E. Scheer, Dr. R. Arnold, Dr. C. Bacca, Dr. T. Böhler, Dr. J. Grebing, Dr. P. Konrad, Dr. V. Kunej, Dr. N. Kang, Dr. H.-F. Pernau, Dr. C. Schirm FB Physik, Universität Konstanz Universitätsstraße 10, 78457 Konstanz 


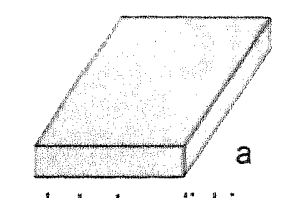

substrate polishing

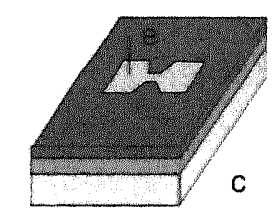

electron beam exposure

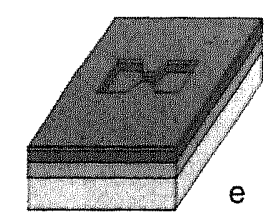

metal deposition
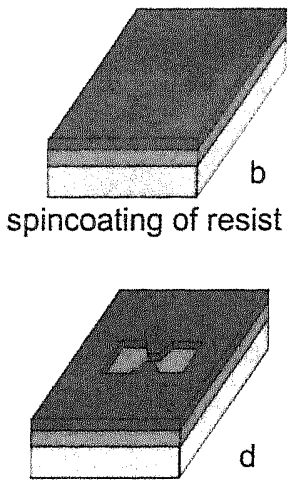

development

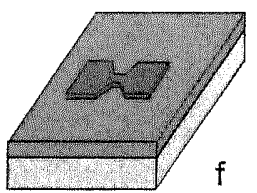

"Lift-off"

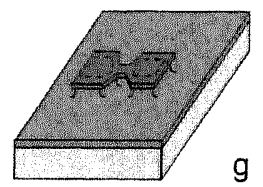

plasma etching

Fig. 2. Lithographic sample propiration procedure.

lirectly on the polyimid according to the lithographically defined structure remains. By reactive ion-etching in an oxygen plasma, part of the polyimid layer is etched away in the end. The etching process is isotropical, getting underneath the metal edges a little, and thus the constriction becomes totally underetched and can be made free-standing. The length of the free-standing part can be altered by varying the etching time and will later determine the displacement ratio when bending the sample. Roughly $0.5 \mathrm{~mm} 2$ big contact pads a distance of $1 \mathrm{~cm}$ apart can be connected to the cables of the measurement setup by contact needles, silver paint or soldering tin. For materials that get inhomogeneous (grainy) when deposited by evaporation like zink, instead of the lithographic process, nano-bridge samples are made by growing whiskers

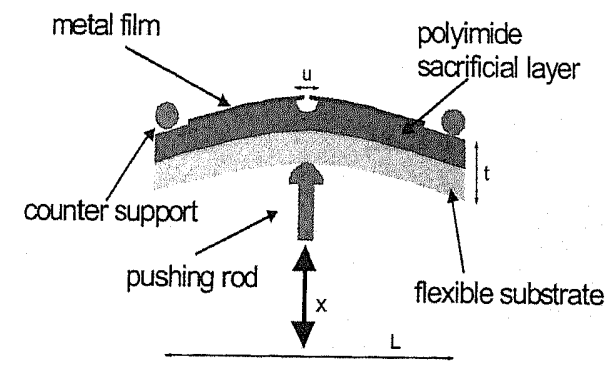

Fig. 3. Bending mechanism.

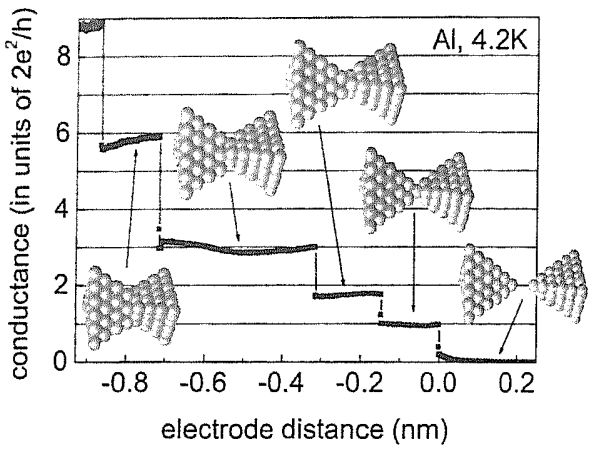

Fig. 4. Opening curve of Al-break-junction. The steproise thinning of the contact is indicated schematically. (Courtesy of E.Scheer)

and positioning them over two gold pads, to which they are then fixed and electrically connected with a silver resin.

The sample is put into the bending mechanism between two counter-supports and a piston with the free-standing nanobridge centered above the latter. By advancing the piston the substrate is bent, the nano-bridge is torn apart, such that it gets thinner and thinner and finally breaks. By retracting the piston and releasing the stress on the substrate, the ends of the contact can be joined together again. The bridge can be opened and closed many times. The displacement ratio is given by the thickness $t$ of the sample, the length $u$ of the free-standing metal-bridge and the distance $L$ of the counter-supports by $d u / d x=6 t u / L 2$. With $L$ on the order of a $\mathrm{cm}$ and $t$ in the sub-mm range, it is sufficient to control the motion of the piston to an accuracy in the $\mu \mathrm{m}$-range in order to change the length of the constriction on scale of $\AA$. A great part of the experiments is made in cryostats at low temperature in order to avoid that thermal excitations smear out the quantum-mechanical effects to observe. For a full characterization of a contact, currentvoltage-curves in the super-conducting state are furthermore required. The normal-conducting state is then reestablished by applying an overcritically large magnetic field. The quantum nature of the conductance through few-atom contacts, however, is so strong and fundamental that especially for the inert gold, it is also observed at room temperature. For so-called opening and closing curves, the resistance (conductance) is monitored while moving the piston. For a fixed more or less bent position of the sample also the current-voltage-characteristic for one contact configuration at a time can be measured.

\section{Conductance Quantization}

While pulling apart the contact a step-like decrease in the conductance is observed. The steps are understood as rearrangements of the atoms in the contact region, while in between under elastic deformation the conductance stays nearly constant. When the contact finally breaks off, there is an exponentiel decay of the conductance according to a tunnel barrier quickly increasing its width. Individual opening and closing curves are not reproducible, but the plateaus are 


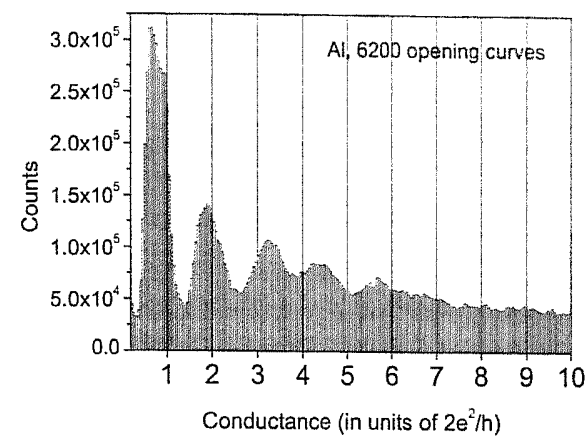

Fis. 5. Conductance histogram for aluminium, from. [1]

preferably found at material specific values, as can be concluded from conductance histograms over many opening and closing cycles. This is attributed to repeatedly realized very stable atomic configurations in the contact region. The peaks in the conductance histograms are neither found at integer multiples of the conductance quantum $G_{0}=2 \mathrm{e}^{2} / h$, nor do they appear at regular intervals. Their positions are, however, element specific. ${ }^{[2]}$ This is explained through the fact that several conductance channels are contributing, each one of which has a transmission probability between zero and one. The number of conduction channels changes with the conductance jumps, but stays constant on the plateaus.

Through a waveguide a number of modes are transmitted according to its cross-section. We regard the example of a two dimensional electron gas (2DEG), that by gate electrodes is further confined to width $D$ on the length of the waveguide. The wavevector in this direction is then restricted to the discrete values $\kappa x=n \pi / D$. Because electrons are supplied with at most the Fermi energy EF, these modes can be occupied up to a critical $n=N_{c}$. Each mode contributes $2 e^{2} / h$ to the conductance $G$ (2 for possible spin states) which is then

$G=\sum_{n=1}^{N_{c}} 2 \frac{e^{2}}{h}=N_{c} \cdot \frac{e^{2}}{h}$

When the width $D$ is changed, the conductance jumps by amounts of $2 e^{2} / h$, each time a new mode enters when its energy falls below EF. This model astonishingly in the case of a $2 \mathrm{DEG}$ still holds true for a quantum-point like short constriction. ${ }^{[3]}$

Tabel 1. Peak positions in conductance histograms (in units of GO) for different materials.

\begin{tabular}{|c|c|}
\hline $\mathrm{Au}$ & $0.9 \quad 1.84-5$ \\
\hline $\mathrm{Al}$ & 0.8193 .24 .3 \\
\hline $\mathrm{Pb}$ & 1.8 \\
\hline $\mathrm{Zn}$ & $072-3$ \\
\hline $\mathrm{Nb}$ & 2.4 \\
\hline $\mathrm{Co}$ & 1.32 .5 \\
\hline
\end{tabular}
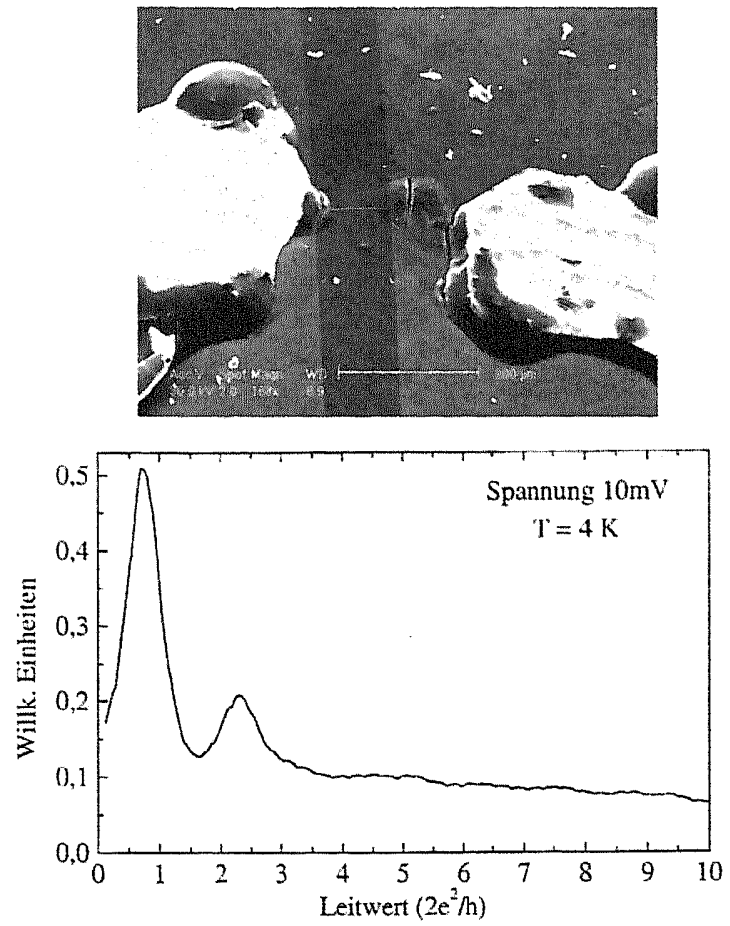

Fig. 6. Image of a Zink-zihisker contacted to gold pads and conductance histogram for Zith-ichisker sample. (Courtesy of $P$. Konrad)

In a mechanically controllable break junction, however, you do not have a link of constant cross-section between to extended solids, but a gradual narrowing from both sides with the smallest diameter only on an atomic length. Transmitted modes are thus not simply defined by laterally quantized and commensurate wave vectors. Nevertheless, however irregular the shape of the constriction may be, we can assume that, at a sufficient distance from it, there is a series of $\mathrm{m}$ incoming electron states on one side and a series of $n$ outgoing states on the other side. The eigenvalues $\tau_{i}$ of $t^{t} t$, where $t$ means the
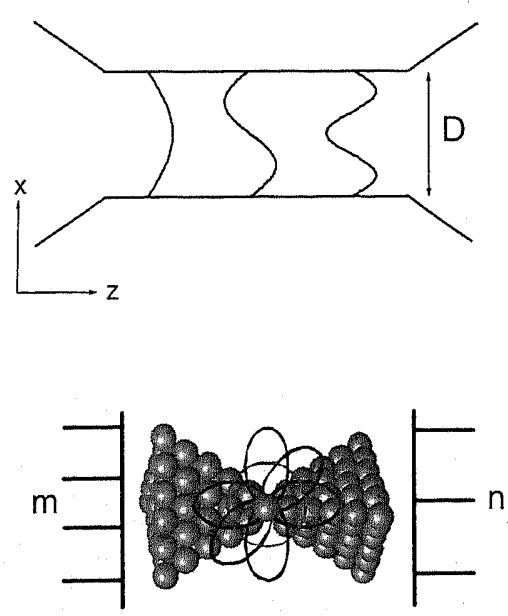

Fig. 7. Waveguide with transmitted standing-wave modes in comparison to atom-contact geometry with $m$ incoming and $n$ outgoing modes and orbitals of the central atom. 
transier matrix fum? an be identified with the transport channels. A few $T_{\text {are }}$ in general sufficient for the description of a contact. Because the problem is diagonalized with the ti, their contributions to the conductance just add. The conductance $G$ is given by the Landauer formula:

$G=2 \frac{v^{n}}{b_{2}} \sum_{i=1}^{v} \tau$

How the transmission probabilities of the channel ensembles $|r|$ are dirived from experiment ${ }^{|t|}$ will be explained in the following.

In the normal conducting state only the total conductance $G$, that is $\Sigma \tau_{i}$, can be known from current-voltage-characteristics (IVs). In the superconducting state, however, besides single electron transfers, multiple Andreev reflections (MAR) give additional contributions to the transport, which leads to nonlinearities in the IVs, from which the number and the values of the ti can be inferred. In materials that do not get superconducting by themselves, superconduc-tivity is induced via the proximity effect. For example, a gold nano-bridge can be covered with an aluminium layer; the last central contact when
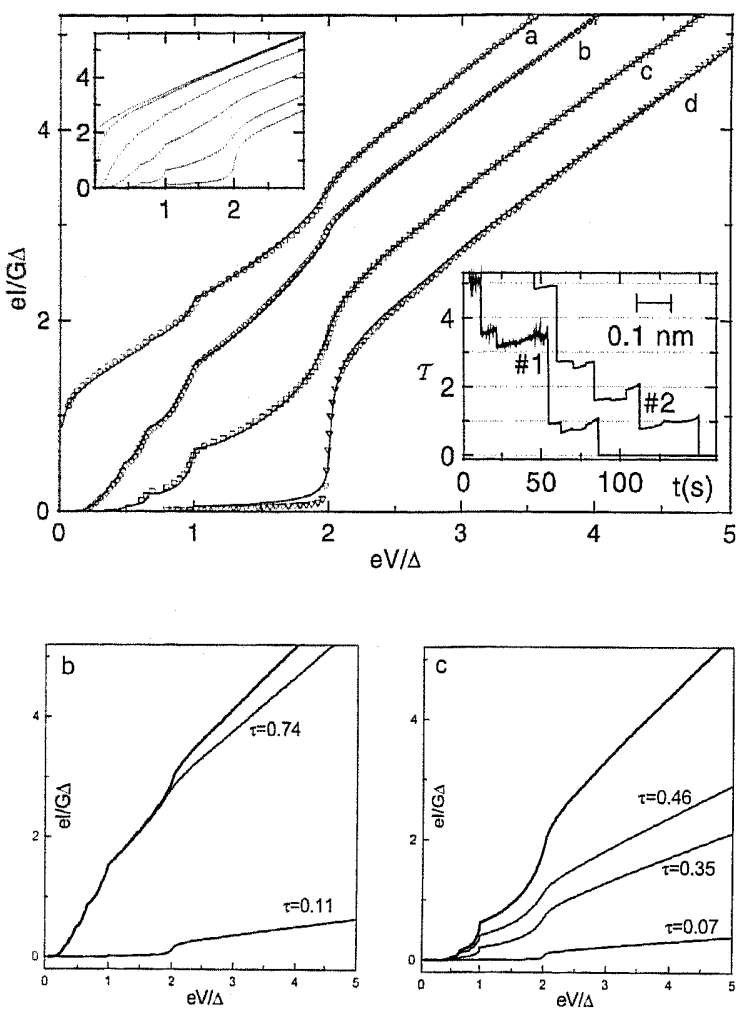

Fig. 8. Superconducting $I V s$ of Al-break-junction in four different opening states, from [6]. Left inset: Theoretical IVs for single channels of transmissions (from bottom to top) $0.1,0.4,0.7,0.9,0.99,1.0$. Right inset: typical opening curves. The total conductances $G$ and channel ensembles (ri) are: a) $G=1.747,10.997,0.46,0.291$, b) $G=0.85,\{0.74,0.11\}$, c) $G=0.88,10.46,0.35,0.07\}$, d) $G=0.025,10.025]$. The calculated repartition of the current onto the channels for curves $b$ and $c$ shows that often one channel is dominating, but a total conductance of equal value can also be made up by several channels of comparable trans-mission.

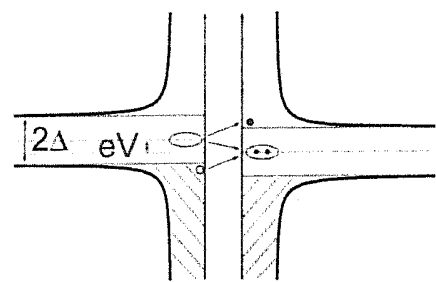

Fig. 9. Example of Andrer' reflection between two super.-conductors with voltage drop $V$ ver the inntion. The rertical axis denotes energy and the thick curces mark the densities of states. In this secont orider process ( $n=2)$ an electron from the reservoir below the $g$ ap and a Cooper pair located at the Fermi energy from the left are transmitted to form a new Cooper pair and a quasi-purticle at an available state aboue the gap on the right.

breaking it will nevertheless consist of gold from the bottom layer. $^{[5]}$

In an Andreev reflection a transmitted electron is reflected as a hole resp. can the process be viewed as the transfer of two electrons that will form a Cooper pair on the other side of the contact. Effectively two elementary charges flow through the contact together. As for break junctions as regarded here, both sides of the contact are superconducting, also higher processes of the kind can take place, as a hole can again be reflected as an electron and so forth. In a MAR of nth order a package of $n+1$ charges is transmitted. The first electron must come from the reservoir below the gap and the last one has to find an unoccupied state above the gap (see Fig. 9). Or a Cooper pair has to be broken to initialize the process. Distributed among $n+1$ charges that pass the voltage $V$ applied over the contact, the energy amount of $2 \Delta$ has to be supplied. That leads to an onset voltage of $2 \Delta /(n+1)$ for the nth order process.

With a transmission probability of less than one for each passage through the contact, higher order Andreev reflections get less probable. But in contrast to tunnel junctions, that are characterized by low transmission, though maybe many such (independent) channels in parallel, quantum point contacts like break junctions have few transport channels, with, however, transmission probabilities not necessarily small compared to one for at least some of them. Therefore MAR here give a nonnegligible contribution to the transport. This is seen in the IVs as an excess current compared to the normal conducting state for voltages $\mathrm{eV}>2 \Delta$ as well as a non-linear rise of the current already at voltages $\mathrm{eV}<2 \Delta$ (subgap-structure). MAR are coherent processes and the rates for the different orders are not independent. Their calculations require quantum-mechanical methods. ${ }^{[7,8]}$ The positions of the step edges nevertheless reflect the onset voltages as derived by the simple argument above. For measured IVs, to get the ensemble $\{\tau \mathrm{i}\}$, a best fit is established as a sum of current-voltage-curves calculated for a series of single $\tau$ (see Fig. 8).

The number of transport channels derived for the last plateaus of opening curves corresponding to one-atom contacts turns out to be material specific and is closely linked to the chemical valence of the respective element. Even if the orbitals of the central atom get modified compared to the free atom because of the embedding in the contact surrounding, a transferred charge has to pass through one of them. Sufficient 
overlap with electron wave functions on both sides of the contact is also required. Monovalent metals like gold, silver or sodium exhibit one transport channel corres-ponding to one s-valence-orbital. sp-metals like aluminium or lead could have four channels, however, only three contribute to the transport. ${ }^{[2]}$ In transition metals like niobium five of the six principally possible channels participate. It is still an open question, to what extent this simple model can be applied to materials with more complex electronic structure as for example half metals like bismuth or antimony, solids for which there are no states directly at the Fermi energy.

\section{Transport Through Magnetic Materials, Clusters, DNA and Double-Contacts}

For magnetic materials the question arises what role electron spin plays and thus whether a magnetic field can block conductance channels or reduce their transmission because only one spin state can be transported. Untiedt et al. ${ }^{[9]}$ do not observe a difference in the conductance histo-grams for cobalt and nickel break junctions with and without magnetic field. It is also still an open question if the ferromagnetic central part of a break junction can be made sufficiently small to induce superconductivity by e.g. aluminium leads in order to determine the transport channel ensemble of for example a cobalt one-atom contact from Andreev reflections through it, or whether these will in principle be suppressed in a superconductor-ferromagnet-superconductor junction. For the transmitted electrons to form Cooper pairs in MAR, equal numbers of opposite spin are required.

The problem in the investigation of transport through magnetic materials is that resistance variations can have other reasons than the lifting of the spin-degeneracy of transport channels. The micromagnetic configuration in the contact region probably changes with the applied magnetic field due to a spatial dependence of the coercive field. A multidomain
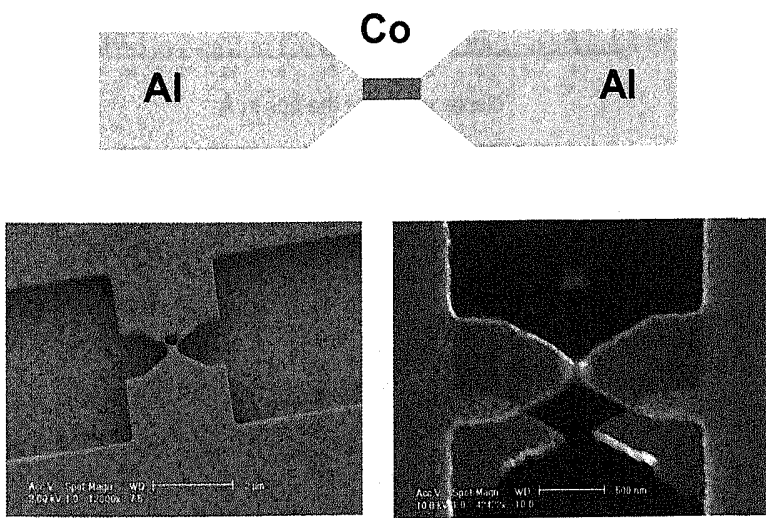

Fig. 10. Scheme of break-junctions with cobalt central part and aluminium leads. Electron microscope images of resist mask and finished metallic structure after lift-off. With a shifted little hole instead of an all-through connected constriction the same mask can be used at normal and oblique incidence for evaporation of both metals. (Courtesy of C. Bacca)

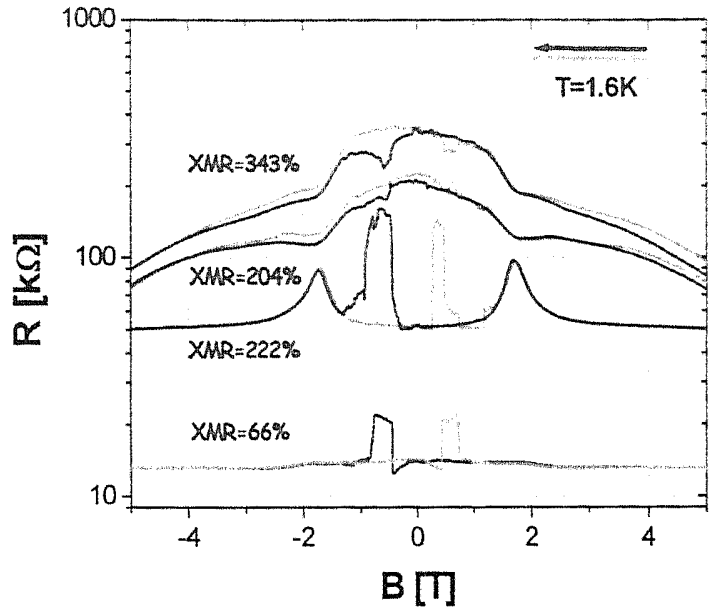

Fig. 11. Resistance zariations in magnetic field sweeps of symmertric all-colalt breakjunctions in the contact (lower curce) and tumeling regime (three upper curves). The $X M R$ parameter is definet as ( $R$ max $-R B=5 T) / R B=5 T$ (Courtesy of $C$. Baca).

structure is likely for the constriction with high surface-tovolume ratio. Resistance recordings for magnetic field rampings show hysteretical features as well as fixed structures which are independent of the field sweep direction (Fig. 11). The influence of the surroundings of the actual point contact is demonstrated on samples prepared on purpose with different opening angles of the cones on both sides of the constriction (Fig. 12). In this case, different coercive fields are expected to saturate magnetic alignment in an applied field on either side of the contact, which leads to clear hysteresis of the resistance in magnetic field sweeps. Magnetostriction cannot be excluded to influence the dependence of the resistance on an external magnetic field. However, samples with non-freestanding break junctions show resistance variations with magnetic field of smaller amplitude, but similar shape as underetched contacts.

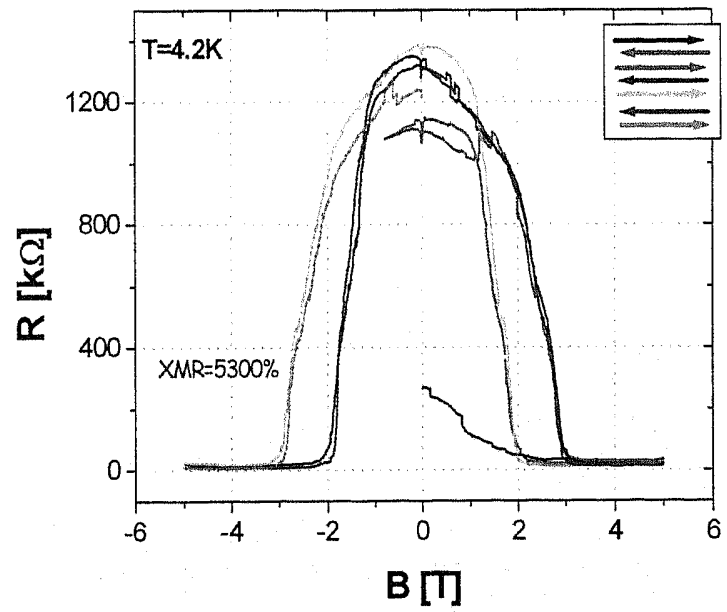

Fig. 12. Purely hysteretical resistance changes with magnetic field of asyntmetric breakjunction. (Courtesy of C. Bacca) 


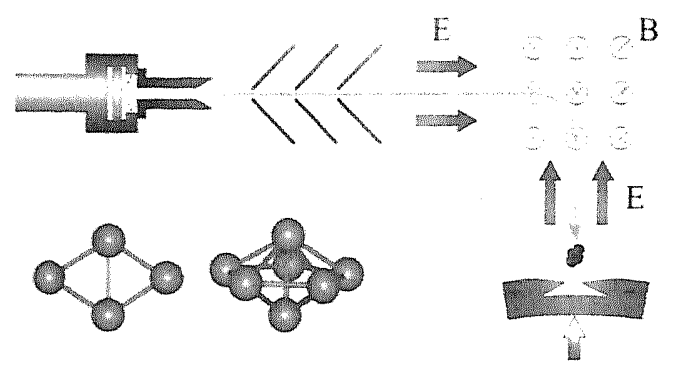

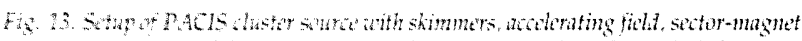

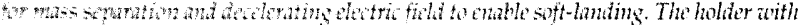

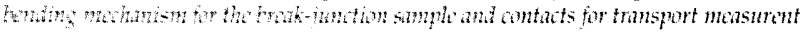

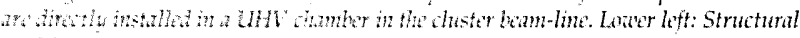

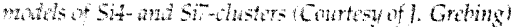

In the fully opened state break junctions present an ideal pair of electrodes a few nanometers or less apart. If molecules or clusters are placed freely suspended between the contact ends, current transport through them can be investigated avoiding the influence of a substrate surface in direct contact. The Pulsed-Arc Cluster-Ion Source (PACIS) in Konstanz can supply silicon clusters of different sizes which we aim to catch in metallic break junctions. After acceleration and mass separation in a sector-magnet, the clusters are slowed down again by electric fields in order to enable a soft landing on the sample without destroying them (Fig. 13). The transport properties will depend not only on the electronic levels of the molecule or cluster, which may get modified by the contacts, but also on the electronic structure of the leads. Especially for Si4-clusters an IV with negative slope $\mathrm{dI} / \mathrm{dV}$ in some voltage range is predicted for aluminium leads whereas a non-linear IV, however no negative differential resistance is expected for gold leads. Si7-clusters should show no such qualitatively different behaviour with these two lead materials. ${ }^{[10]}$

Other than silicon, $\mathrm{C}_{60}$-clusters can be deposited onto the sample by thermal evaporation which is done in situ in the UHV chamber for transport measurements. In comparison. with pure gold break junctions, samples with 0.5 monolayers $\mathrm{C}_{60}$ on top exhibit no last plateau in the opening curves, but an irregular decrease of the conductance down to zero (Fig. 15). A conduc-tance histogram at room temperature shows a broad accumulation of high counts for all conductance values up to about $2 G_{0}$ (Fig. 16). Smaller peaks at higher conductances
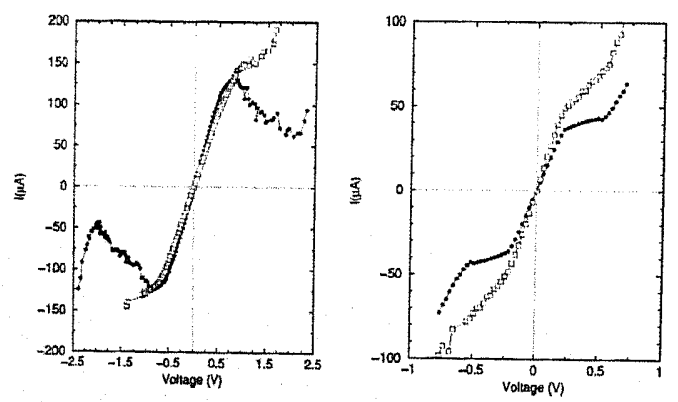

Fig. 14. Calculated IVs for Si4-(full circles) and Si7-clusters (open squares) between aluminium (left) and gold (right) electrodes, from [10]. resemble the signature of pure gold junctions. At low temperature a very significant difference in the histograms has not yet been found. IV-curves of gold break junctions with $\mathrm{C}_{60}$ in comparison to pure gold samples show non-linear behaviour already at voltages below $-100 \mathrm{mV}$. An alternative approach for sample fabrication also pursued consists in making the whole break junction out of $\mathrm{C}_{60}$, of which then a thick layer is evaporated on top of gold ${ }^{[11]}$ or even as only conducting layer.

The investigation of electrical transport through DNA is important to understand DNA damage and repair in biological systems, to build DNA-based electrochemical sensors and to possibly implement DNA as building blocks in electronic devices. Break junctions are especially suitable contacts for conductance measurements as they allow a controlled streching of the helix molecules. Discrete resistance jumps are observed while opening or closing a break junction with DNA suspended between the contact ends (Fig. 18), which may be due to conformational changes in the DNA or the contacts as well as electrical charge transfer and localization in the molecule. Current-voltage characteristics show non-linear behaviour (Fig. 19).

A series of two contacts, as for a cluster or a molecule suspended between two electrodes, can also be formed by two metal-to-metal contacts, for example one being a break junction and the other a tunnel junction defined by a thin oxide layer, such that a metallic island results between the contacts. Even if
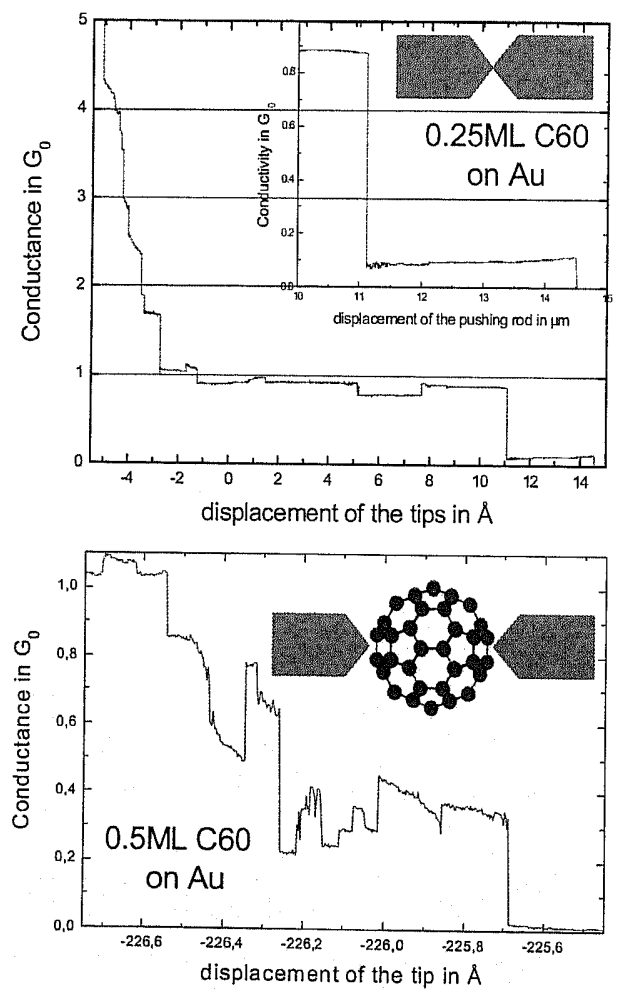

Fig. 15. Opening curve of gold break-junction samples taken at room temperature with 0.25 monolayers of $C 60$ evaporated on top, where probably no cluster sits in the junction, and 0.5 monolayers, where transport probably is through C60. (Courtesy of T. Böhler) 

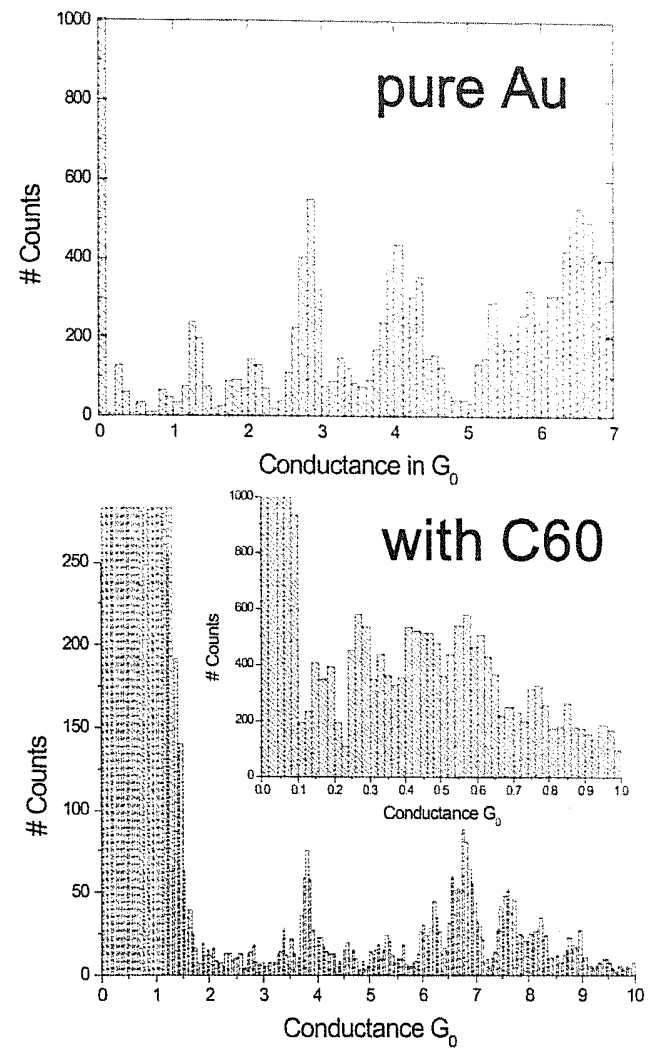

Fig. 16. Conductance histograms at room temperature of gold break junction with $\mathrm{C} 60 \mathrm{in}$ comparison to pure gold sample. (Courtesy of T. Böhler)

the island is of $\mu \mathrm{m}$-size, its capacitance $C$ with respect to the leads can be so small that single-electron effects become recognizable. A minimum energy of $E_{c}=\mathrm{e}^{2} / 2 \mathrm{C}$ is required for the transfer of even only one electron onto the island, such that current flow can only set in at a finite voltage. (That the current onset in the normal state is at a voltage corresponding to $\mathrm{eV}=2 E_{\mathrm{c}}$ (Fig. 21) is due to the fact that the applied voltage is reparted over both contacts.) Also a single or only a few charges on the island will shift its electric potential with respect to the leads such that no further electrons may pass onto it. These phenomena are called Coulomb-blockade and lead to staircase-

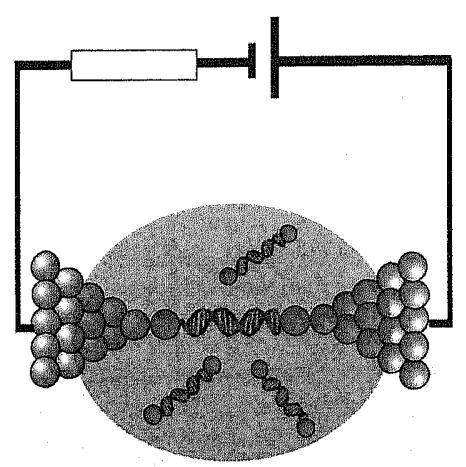

Fig. 17. Scheme of DNA in break junction circuit obtained by drying a droplet of solution on the sample. (Courtesy of N. Kang)
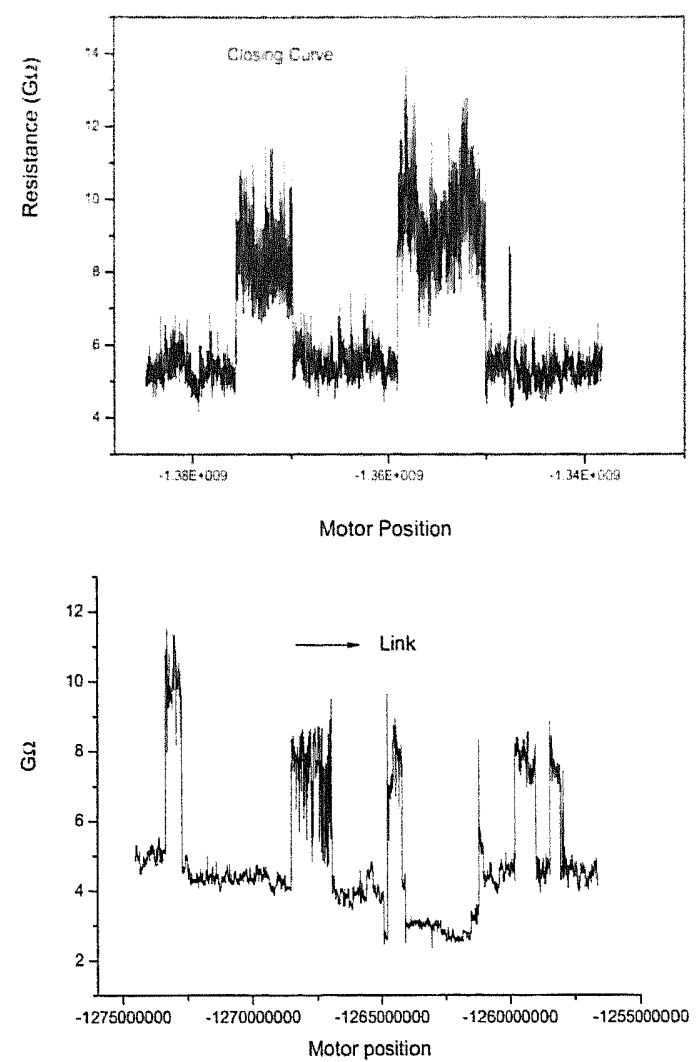

Fig. 18. Discrete resistance jumps obsered while closinglopening a break junction with DNA. (Courtesy of N. Kang)

like nonlinear IV-characteristics. (Of course, a metallic island of $\mu \mathrm{m}$-width and -length and $\sim 100 \mathrm{~nm}$ thickness contains many conduction electrons; by "charges" we here only refer to the few in excess to neutrality.) The island potential may further be influenced via a gate electrode, a possibility which we will not regard further in the short presentation of the issue to discuss here.

The double contact setup with gate is well known for two tunnel contacts as the single-electron transistor. In this case as well as even with contacts of higher transmission in the normal conducting state, where current consists of a single kind of carriers, the IV shows regular Coulomb steps (Fig. 21). If, however, at least one of the contacts has transport channels of not too low transmission, in the superconducting state, multiple Andreev reflections come into play. But can these multiplecharge package transfers persist when charge accumulation on the island leads to Coulomb blockade? Calculations of the IV characteristics have been carried through based on different assumptions. If a package of $n+1$ charges in fact has to be transmitted as a whole, following an orthodox theory (OT) ${ }^{[12]}$ model, the transport voltage has to supply the energy to overcome the gap as well as the full charging energy leading to the condition $\mathrm{VV}>2(n+1) E_{\mathrm{c}}+4 \Delta /(n+1)$ (for equal capacitances of the two contacts, vanishing gate voltage and initially neutral island). If simultaneously to MAR over one contact, there can be tunneling or even also MAR processes over the other contact, 

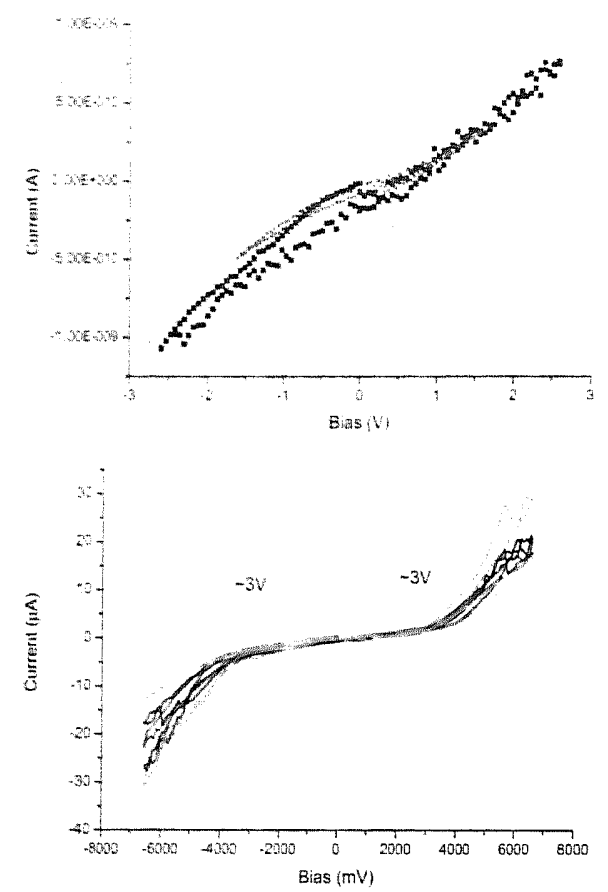

Fis. 19. Examples of $\mathrm{H}$ cures for DNA showing non-linear behawiour. (Courtesy of A. King

not all $n+1$ charges need to accumulate on the island at once. A quantum-mechanical Green's functions model for the combination of a tunnel junction with a second contact of higher transmission predicts current onset at considerably lower voltage and a different step pattern in the IV then OT (Fig. 22). Assuming coherence over the whole island length and including multiple reflection processes extending over both junctions in the Green's functions calculations results in a lower current as well as shifts steps in the IV and the current onset to slightly higher voltages. ${ }^{[13]}$ Even in the normal state, coherent multiple reflections over both junctions would alter the current in magnitude compared to the case where the passage across the island links transport processes through both contacts only incoherently (Fig. 21).

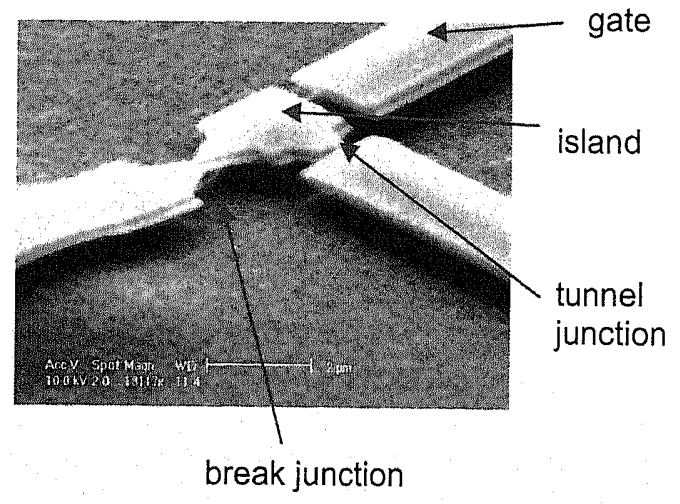

Fig. 20. Lithographically made circuit with Al break junction, Al-island and tunnel junction $(A l, A l$-oxid, Al) in series as well as gate electrode.

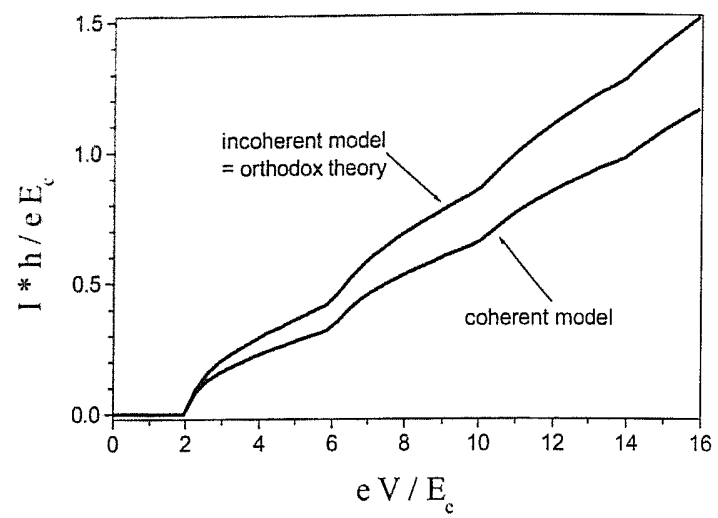

Fig. 21. Calculated IV for double junction in the normal conducting state for transmissions of the two links 0.06 and 0.48 respectively and equal capacitances, no gate woltage, following different models.

\section{Summary}

Electrical contacts of the width of only one atom can be realized by the break-junction technique. The conductance decreases stepwise due to structural reconfigurations when tearing a nano-bridge in the few-atom range. Transport is described by an ensemble of channels with possibly quite high transmission probabilities. Therefore transport processes, an important part of which are based on multiple reflections, in a series of junctions should show an interplay of Coulomb blockade and coherence or decoherence effects. For a single break-junction the last one-atom contact consists of a materialspecific channel ensemble, determined by the chemical valance as verified for quite a number of metals. d-electrons in half-metals and spin-effects in magnetic materials will complicate this simple model. Break-junctions also provide ideal contacts to investigate transport through freely suspended clusters or molecules like DNA. Electric circuits with few-atom constrictions or molecular contacts allow to study a

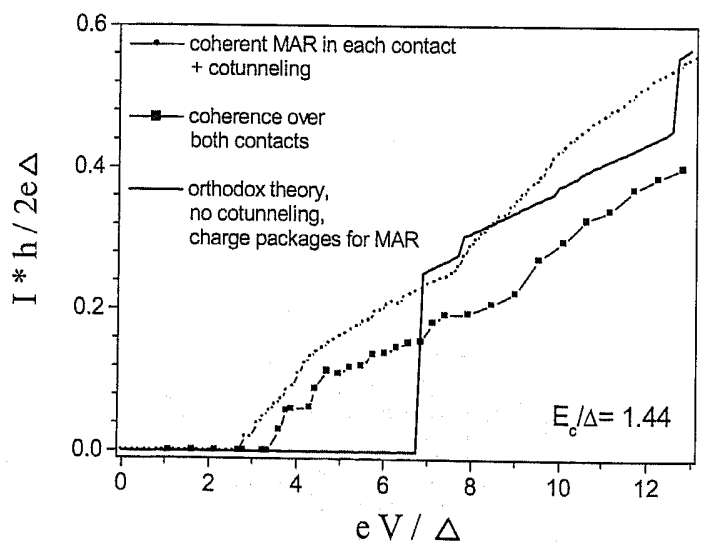

Fig. 22. Calculated IV for double junction with trans-missions 0.06 and 0.48 and equal capacitances in the superconducting state from different models. 
mesoscopic regime where transport becomes governed by quantum-mechanical effects. ${ }^{[1]]}$

Received: $\mathrm{Mny}$ 12, 2005

Final version: Junt 01, 2005

[1] A. I. Yanson, J. M. van Ruitenbeek, Phys. Rev. Lett. 1997, $79,2157$.

[2] E. Scheer et al., Nature 1998, 394, 154.

[3] B. J. van Wees et al., Phys. Rev. Lett. 1988, 60, 848.

[4] E. Scheer et al, Superlattices and Microstructures 1998, 23, 747.
[5] E. Scheer tal. Phys. Rev. Lwt. 2001, 86, 284.

[6] E. Scheer th al, Phys. Rev. Lett, 1997, 78, 3535.

[7] J. C. Cuevas ef al., Phys. Re'. B 1996, 54, 7366.

[8] D. Averin, A. Bardas. Phys. Rev, Letf. 1995, 75, 1831.

[9] C. Untiedt et al., Phys, Ret. B 2004, 69, 081401.

[10] C. Roland et al., Phys, Rev, B 2002, 66, 035332.

[11] T. Böhler et al., Nanotecln, 2004, 15, 465.

[12] H. Grabert, M. Devoret, Single Charge Tunneling, Plenum NewYork 1992

[13] U. Schröter, E. Scheer, submittıt.

[14] N. Agrait et al., Physics Reports 2003, 377,87. 